\title{
A transit timing analysis with combined ground- and space-based photometry
}

\author{
St. Raetz ${ }^{1}$, M. Fernandez ${ }^{2}$, C. Marka ${ }^{3}$, A. M. Heras ${ }^{1}$, and G. Maciejewski ${ }^{4}$
}

1 European Space Agency, ESTEC, SRE-S, Keplerlaan 1, 2201 AZ Noordwijk, The Netherlands: sraetz@cosmos.esa.int

2 Instituto de Astrofisica de Andalucia (IAA-CSIC), Glorieta de la Astronomía, 18008 Granada, Spain

3 Astrophysikalisches Institut und Universitäts-Sternwarte, Schillergäßchen 2-3, Jena, Germany

4 Centre for Astronomy, Faculty of Physics, Astronomy and Informatics, Nicolaus Copernicus University, Grudziadzka 5, 87-100 Torun, Poland

\begin{abstract}
The analysis of timing variations of transiting exoplanets allows one to discover additional planets in the system. Such bodies can be very low in mass and radius, so that they remain undetected by transit or radial velocity methods.

The CoRoT satellite looks back on six years of high precision photometry of a very high number of stars. Thousands of transiting events are detected from which 27 were confirmed to be transiting planets so far. In my research I search and analyze TTVs in the CoRoT sample and combine the unprecedented precision of the light curves with ground-based follow-up photometry. Because CoRoT can observe transiting planets only for a maximum duration of 150 days the ground-based follow-up can help to refine the ephemeris. Here we present first examples.
\end{abstract}

\section{Introduction, observations, data reduction, and analysis}

The transit event is - in a first approximation - a periodic phenomenon. In a system where a known planet transits its host star, a second planet in that system can cause the time between transits to vary. This technique is itself a planet detection method that is very powerful for searching low-mass planets and is most sensitive for planets in mean-motion resonances (e.g. [5]).

The ground-based high-precision photometric follow-up observations of the known transiting planets from the CoRoT sample were carried out at two observatories located in Germany and in Spain.

At the University Observatory Jena (Germany) we used the 60-cm Schmidt telescope equipped with the optical CCD camera STK [6]. Several light curves of our CoRoT-sample were obtained on the Observatorio de Sierra Nevada (OSN, Spain) using the $1.5 \mathrm{~m}$ reflector with a VersArray:2048B CCD camera. All observations were done in the $R$-band.

The photometric data were reduced following standard procedures including subtraction of bias (as overscan, only if available) and dark, and division by a sky flat-field. Magnitudes were derived with differential aperture photometry against an optimum artificial comparison star which is composed of the weighted average of all available field stars [2].

The CoRoT light curves were downloaded from the "NASA Exoplanet Archive". Only the white-light data were used for the determination of the transit times.

The Transit Analysis Package (TAP, version 2.104, [3]) was used to analyze all ground- and spacebased transit light curves. TAP uses a wavelet-based Markov chain Monte Carlo approach and employs the quadratic limb-darkening law to model the distribution of the flux on a stellar disc. 


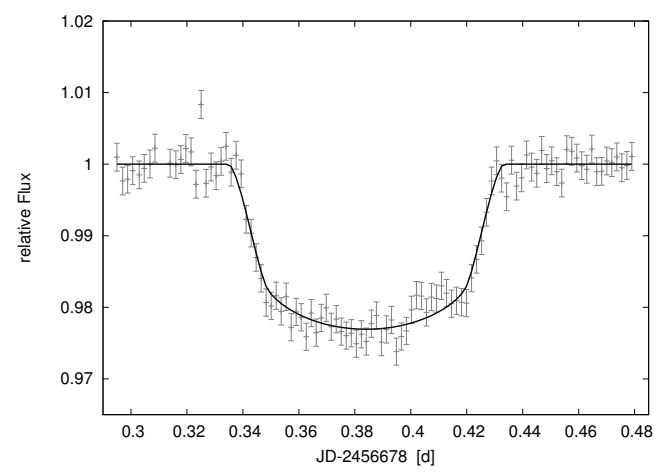

Fig. 1. Light curve of CoRoT-18b that was observed on 2014 Jan. 20th with the $1.5 \mathrm{~m}$ telescope at the Observatorio de Sierra Nevada. It clearly shows a spot signature between 0.40 and $0.42 \mathrm{~d}$.

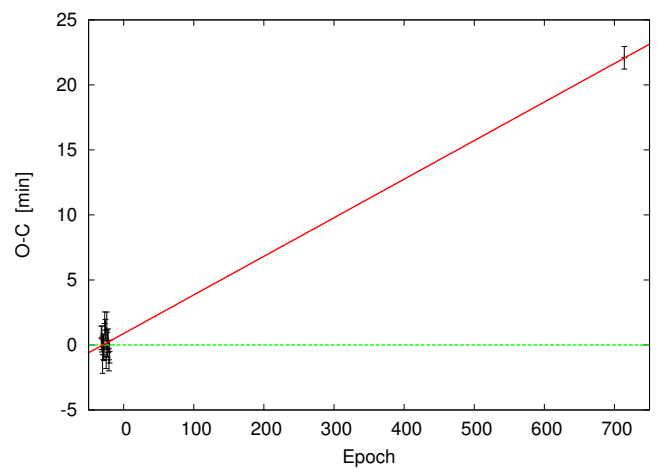

Fig. 2. O-C-diagram for CoRoT-18b. The updated ephemeris and the ephemeris given in [4] are shown as solid red and dashed green lines, respectively

\section{Examples and First Results}

\section{CoRoT-8b}

CoRoT-8b, which was discovered by CoRoT during the first long run in constellation Aquila (LRc01), orbits a $\mathrm{K} 1 \mathrm{dwarf}$ in $\sim 6.2$ days. With a radius of $0.57 \pm 0.02 \mathrm{R}_{\text {Jup }}$ and a mass of $0.22 \pm 0.03 \mathrm{M}_{\text {Jup }}$, CoRoT-8b appears to be somewhat between Saturn and Neptune [1]. One transit was observed on 2012 September 6th with the 60-cm Schmidt telescope of the University Observatory Jena. The transit occurred approximately $1 \mathrm{~h}$ earlier than expected from the ephemeris of [1] (corrected for the known discrepancy in $T_{0}$ ), hence only a partial transit could be observed. This resulted in large timing uncertainties and therefore more observations are needed to better constrain the orbital elements.

\section{CoRoT-18b}

CoRoT-18b was detected in the CoRoT field SRa03 [4]. With a mass of $3.47 \pm 0.38 \mathrm{M}_{\text {Jup }}$ and a radius of $1.31 \pm 0.18 \mathrm{R}_{\text {Jup }}$ it is a massive hot Jupiter that orbits its G9V host star in $\sim 1.1 \mathrm{~d}$.

We observed one transit of CoRoT-18b in January 2014 at OSN. The light curve is shown in Fig. 1. The ground-based light curve as well as the CoRoT ones reveal features that affect the transit shape and could be attributable to starspots. We removed the spot-affected parts from the individual light curves before phase-folding and binning. The best-fit model based on the phase-folded light curve was used as a template and fitted to the individual light curves for which the parameters were allowed to vary under the Gaussian penalty defined by the derived errors.

Fig. 2 shows the final O-C-diagram for CoRoT-18b. The ground-based measurement deviates by $\sim 22 \mathrm{~min}$ from the predictions, and on this basis the ephemeris were refined as:

$$
\begin{array}{r}
T_{\mathrm{c}}(E)\left[B J D_{T D B}\right]=(2455321.72551+E \cdot 1.9000899) \mathrm{d} \\
\pm 0.00018 \quad \pm 0.0000009
\end{array}
$$

\section{References}

1. Bordé, P., Bouchy, F., Deleuil, M., et al., A\&A 520, A66 (2010)

2. Broeg, C., Fernández, M., \& Neuhäuser, R., Astronomische Nachrichten 326, 134 (2005)

3. Gazak, J. Z., Johnson, J. A., Tonry, J., et al., Advances in Astronomy 2012, 697967 (2012)

4. Hébrard, G., Evans, T. M., Alonso, R., et al., A\&A 533, A130 (2011)

5. Holman, M. J., \& Murray, N. W., Science 307, 1288 (2005)

6. Mugrauer, M., \& Berthold, T., Astronomische Nachrichten 331, 449 (2010) 